\title{
Avaliação das necessidades de tratamento odontológico e da capacidade produtiva da rede de atenção básica em saúde bucal no município de Pelotas, estado do Rio Grande do Sul, Brasil, 2009
}

doi: $10.5123 /$ S1679-49742012000200016

\author{
Assessment of dental treatment needs and productive capacity of primary dental care in the \\ municipality of Pelotas, state of Rio Grande do Sul, Brazil, 2009
}

Cleusa Marfiza Guimarães Jaccottet

Secretaria Municipal de Saúde de Pelotas-RS, Brasil

Programa de Pós-Graduação em Epidemiologia, Universidade Federal de Pelotas, Pelotas-RS, Brasil

Aluísio J. D. Barros

Programa de Pós-Graduação em Epidemiologia, Universidade Federal de Pelotas, Pelotas-RS, Brasil

Maria Beatriz Junqueira de Camargo

Programa de Pós-Graduação em Epidemiologia, Universidade Federal de Pelotas, Pelotas-RS, Brasil

Andreia Morales Cascaes

Programa de Pós-Graduação em Epidemiologia, Universidade Federal de Pelotas, Pelotas-RS, Brasil

\section{Resumo}

Objetivo: descrever as necessidades de tratamento odontológico da população e a capacidade produtiva da atenção básica no município de Pelotas, Rio Grande do Sul. Métodos: foram utilizados dados do inquérito de saúde bucal de 2003 para estimar indicadores de necessidade de restaurações, extrações e tratamento periodontal entre indivíduos com 15 anos ou mais de idade, segundo faixa etária e renda. 0 potencial produtivo foi estimado a partir dos dados do Sistema de Informações Ambulatoriais (SIA/SUS). Resultados: mais de 234 mil indivíduos tinham necessidade de tratamento, incluindo 274.085 elementos dentários necessitando de restauração, 107.659 de extração, 282.986 sextantes necessitando remoção de cálculo e 17.803 de tratamento periodontal. Assim, seriam necessárias mais de 680 mil consultas clínicas, em grande desproporção com a produção ambulatorial do município no ano de 2008, de 47.179 procedimentos. Conclusão: o potencial produtivo do serviço odontológico era deficitário para atender a todas as necessidades da população.

Palavras-chave: Atenção Primária à Saúde; Necessidades e Demandas de Serviços de Saúde; Assistência Odontológica Integral; Saúde Bucal.

\begin{abstract}
Objective: to describe dental treatment requirements and productive capacity of primary health care in the municipality of Pelotas, Brazil. Methods: This study used data from the 2003 National Oral Health Survey to estimate the indicators of individuals 15 years or above, that needed restorations in the teeth, extractions, and periodontal treatment, by age and income. The productive potential of the city was estimated using data from the Ambulatory Care Information System/National Health System. Results: over 234,000 individuals showed a need of dental treatment; 274,085 needed teeth restoration; 107,659 needed extraction; 282,986 sextants with needs of calculus removal; and 17,803 needed periodontal treatment. The 680,000 dental visits that were necessary are hugely disproportional compared with the 47,179 procedures performed in the municipality in 2008. Conclusion: productive capacity of the public oral health care in Pelotas-RS was far from being sufficient to cater to all the needs of the population.
\end{abstract}

Key words: Primary Attention; Health Services Needs and Demand; Comprehensive Dental Care; Oral Health.

Endereço para correspondência:

Rua Quinze de Novembro, 1054/202, Centro, Pelotas-RS, Brasil. CEP: $96015-000$

E-mail: cleusajaccottet@hotmail.com 


\section{Introdução}

A saúde bucal é essencial para o bem estar do indivíduo. A despeito disso, grande parcela da população encontra-se desassistida nessa área. ${ }^{1}$ No Brasil, o modelo de atenção em saúde bucal, baseado na abordagem cirúrgico-restauradora e hegemônico durante décadas, apresentou poucos avanços no quadro epidemiológico. Historicamente, as políticas de saúde bucal voltadas para a população de escolares ${ }^{2} \mathrm{e} 0$ atendimento de urgência para adultos ${ }^{3}$ caracterizavam-se pela inexistência de integração com os demais setores da Saúde.

Com a inserção das equipes de saúde bucal na Estratégia Saúde da Família (ESF) em 2000, o Sistema Único de Saúde (SUS) prevê a evolução do modelo assistencial centrado na doença e demanda espontânea para o modelo de atenção integral à saúde, no qual devem-se articular ações de promoção e proteção ao lado daquelas de recuperação propriamente ditas. ${ }^{4}$ Além da implantação das equipes de Saúde Bucal, o lançamento do programa 'Brasil Sorridente' e a publicação da Política Nacional de Saúde Bucal têm possibilitado a ampliação do acesso aos cuidados em saúde bucal no país. ${ }^{5}$ Estima-se que, de 2003 a 2008, o governo federal tenha investido, aproximadamente, 2,4 bilhões de reais nos serviços e ações de saúde bucal. ${ }^{6}$ Em 2009, o Brasil contava com 18.982 equipes de saúde bucal, distribuídas por 4.717 municípios. $^{7}$

\section{No Brasil, o modelo de atenção em saúde bucal, baseado na abordagem cirúrgico-restauradora e hegemônico durante décadas, apresentou poucos avanços no quadro epidemiológico.}

Segundo dados da Pesquisa Nacional por Amostra de Domicílios, desenvolvida pela Fundação Instituto Brasileiro de Geografia e Estatística (PNAD/IBGE), o percentual de brasileiros que nunca consultou um dentista caiu de 18,7\%, em 1998, para 15,9\%, em $2003 .{ }^{8}$ Essa redução pode estar relacionada à maior oferta de serviços, não refletindo, necessariamente, melhoria da qualidade dos atendimentos. Na maioria das vezes, a expansão da oferta de serviços ocorre sem planejamento e programação das atividades e a prática profissional continua atrelada a uma demanda reprimida e crescente de atendimento cirúrgico-restaurador, sem perceber melhorias nas condições de saúde da comunidade. ${ }^{9}$ Ademais, as desigualdades no acesso e na utilização dos serviços entre os grupos de renda são evidentes. A proporção de adultos que nunca foi ao dentista chega a ser 16 vezes maior no quintil de renda mais pobre, comparativamente ao quintil mais rico, e na população jovem de 7 a 19 anos, essas proporções já são marcantes: $41,0 \%$ de jovens no quintil de renda mais baixa; e 3,4\% deles no quintil de renda mais elevada. ${ }^{10}$

O diagnóstico das condições de saúde bucal e das necessidades de tratamento da população, bem como a avaliação do modelo de atenção em saúde vigente, é de fundamental importância como primeiro passo no sentido da programação e planejamento em saúde bucal, permitindo estabelecer prioridades de ação e alocação de recursos de forma direcionada à melhoria das condições de saúde da população.

0 presente estudo teve por objetivo estimar as necessidades de tratamento de dentes permanentes na população com 15 anos de idade ou mais, além de comparar as necessidades estimadas com o potencial produtivo da rede de Atenção Básica em Saúde Bucal no Município de Pelotas, Estado do Rio Grande do Sul, Brasil.

\section{Métodos}

O município estudado, Pelotas-RS, contava uma população de cerca de 340 mil habitantes em 2007. ${ }^{11}$ 0 sistema de saúde foi municipalizado no ano 2000 e atualmente, a rede de Atenção Básica, composta por 50 unidades básicas de saúde (UBS), conta com 29 equipes de Saúde da Família para cobrir uma população aproximada de 100 mil pessoas. A saúde bucal não se encontra inserida na Estratégia Saúde da Família - ESF -, adotando um modelo eminentemente assistencial. 0 atendimento odontológico está implantado em 36 UBS, 26 na zona urbana e dez na zona rural, e conta com 66 cirurgiões-dentistas, cinco deles lotados em escolas e quatro em cargos administrativos.

Para estimar as necessidades de tratamento odontológico em Pelotas-RS, foram utilizados dados do município obtidos pelo 'Levantamento das Condições 
de Saúde Bucal da População do Rio Grande do Sul' em 2003 (SB/RS 2003), além de dados do IBGE. 0 SB/RS 2003 é parte de um inquérito nacional e incluiu uma ampliação da amostra original. Maiores informações sobre a metodologia desse estudo estão disponíveis no relatório final do SB-Brasil. ${ }^{12}$

No inquérito, foram estudados os grupos etários de 18-36 meses e de 5, 12, 15-19, 35-44 e 65-74 anos. No presente estudo, foi analisada a população a partir dos 15 anos de idade, de quem houvesse informação de renda, estimando-se as necessidades de tratamento restaurador, extrações e tratamento periodontal.

Para a avaliação de necessidades de restaurações e extrações dentárias, foi utilizada uma estratégia constituída de dois passos. Em primeiro lugar, estimou-se o percentual de indivíduos com alguma necessidade, para cada idade. A seguir, e apenas para o grupo com necessidade, estimou-se o número médio de dentes que necessitavam de tratamento, também por idade. Ao final, estimou-se o número total de indivíduos e de elementos dentários com necessidade de tratamento. Para a avaliação de necessidades de tratamento periodontal, foi realizado processo semelhante, embora a avaliação fosse realizada por sextante.

As necessidades de tratamento para cárie dentária, estimadas a partir de dados do SB/RS 2003, foram classificadas como 'tratamento restaurador de uma ou mais superfícies' e 'extração dentária'.

Em relação às doenças periodontais, as necessidades de tratamento foram estimadas com base nos dados de morbidade, de acordo com o número de sextantes apresentando cálculo dental e bolsa periodontal a partir de $4 \mathrm{~mm}$. Para necessidade de tratamento periodontal, optou-se por não incluir o sangramento gengival, por ser essa uma condição sensível às medidas de promoção e prevenção em saúde bucal, passível de ser executada por profissionais auxiliares com supervisão do cirurgião-dentista e não exigindo, necessariamente, tratamento clínico. ${ }^{13}$

A população foi dividida em quintis de renda familiar e a estratégia aqui descrita foi aplicada separadamente, para dois grupos: os 40,0\% de menor renda; e os $60,0 \%$ de maior renda. 0 s $40,0 \%$ da população com menor renda familiar foram tratados integralmente, como usuários do serviço público. Dos $60,0 \%$ restantes, considerou-se $20,0 \%$ deles usuários de serviços privados, com base nos dados da Agência Nacional de Saúde Suplementar (ANS) 14 que estimam em $18,0 \%$ a população do Estado do Rio Grande do Sul coberta por planos privados de saúde. Assim, aplicou-se redução de 20,0\% nas necessidades estimadas para esse grupo.

Como os dados do SB/RS 2003 são limitados a algumas faixas etárias, não é possível estimar diretamente as necessidades para todas as idades, razão porque foram utilizados modelos de regressão com polinômios fracionais, para interpolar as estimativas de necessidade dos grupos sem informação. Essa estratégia inclui uma família de curvas muito mais numerosa, permitindo o ajuste adequado ao modelo. ${ }^{15} \mathrm{Os}$ modelos foram ajustados com as necessidades como desfecho, e a idade em anos inteiros como preditor, separadamente para os $40,0 \%$ de menor renda e os $60,0 \%$ de maior renda.

Dados demográficos do município referentes ao número de habitantes para cada grupo etário foram extraídos do sistema de consultas SIDRA (Sistema IBGE de Recuperação Automática), com base no Censo Demográfico de 2000, realizado pelo IBGE, ${ }^{16} \mathrm{e}$ utilizados com o intuito de expandir as necessidades de tratamento da amostra para o total da população do município.

A estimativa de necessidades de tratamento odontológico da população estudada em Pelotas-RS foi realizada em duas etapas:

- primeiramente, estimou-se o percentual de indivíduos da amostra com necessidade de tratamento: utilizou-se a amostra total para o Estado do Rio Grande do Sul, visto que as análises comparativas realizadas junto ao banco de dados SB/Brasil-RS 2003 entre a macrorregião de Pelotas-RS, de cuja amostra o Município fez parte, e os dados do Estado apresentaram resultados bastante semelhantes; a utilização da amostra do Estado (N=24.988) e não apenas da subamostra da macrorregião de PelotasRS ( $\mathrm{N}=2.411)$ implica um ganho substancial de precisão nas estimativas;

- segundamente, as proporções estimadas foram aplicadas à população total do Município, a partir de dados do Censo 2000.

Para o cálculo do potencial produtivo de Pelotas-RS, utilizou-se o banco de dados do Sistema de Informações Ambulatoriais, do Sistema Único de Saúde (SIA/ SUS),${ }^{16}$ de onde foram retirados os dados de produção do atendimento odontológico ambulatorial no município. Os dados utilizados no estudo referem-se à produ- 
ção ambulatorial local no ano de 2008 e contemplam o número e o tipo de procedimento odontológico realizado pela rede de Atenção Básica. 0 banco de dados do Cadastro Nacional dos Estabelecimentos de Saúde (CNES) ${ }^{17}$ foi utilizado para a obtenção dos dados de infraestrutura do município e equipes odontológicas: número de equipamentos; e número e tipo de profissionais (cirurgiões-dentistas, técnicos em saúde bucal, auxiliares de saúde bucal). Essas informações foram utilizadas para avaliar a capacidade instalada da rede municipal: número de UBS com consultórios odontológicos; unidades que prestam atendimento especializado; e número de cirurgiões-dentistas disponíveis para atendimento. Informações junto à Secretaria Municipal de Saúde de Pelotas-RS foram consultadas para checar possíveis divergências entre os dados do CNES e a situação encontrada no nível local, além de informações sobre o gerenciamento do serviço, turnos de atendimento odontológico efetivo segundo tipos de programas oferecidos.

0 cálculo do potencial produtivo da rede de Atenção Básica em Saúde Bucal foi avaliado de acordo com os parâmetros estabelecidos pelo Departamento Nacional de Auditoria do SUS (Denasus)/Ministério da Saúde, que estabelece o número de consultas por turno de atendimento. ${ }^{18} 0$ Denasus/MS define um número de três atendimentos/hora/profissional, com tempo médio de 20 minutos e média de 22 dias úteis/mês. Portanto, a fórmula do cálculo do potencial produtivo foi: $\mathrm{n}^{0}$ de turnos de atendimento $\times$ carga horária diária $\times 3$ consultas/hora $\times 22$ dias úteis/mês.

O Ministério da Saúde recomenda que 75,0 a 85,0\% das horas contratadas devem ser dedicadas à assistência, e o restante do tempo utilizado para outras atividades.4 Como o serviço de saúde bucal de Pelotas-RS não preconiza a redução da carga horária para essas atividades, nem possui pessoal auxiliar, o cálculo foi realizado de acordo com a situação real, alocando a carga horária integral para atividades clínicas.

As necessidades de tratamento da população estimadas neste estudo foram comparadas com os dados de produção ambulatorial do município e com o potencial produtivo da rede de Atenção Básica em Saúde Bucal, o que possibilitou concluir o tempo necessário para a realização de todos os procedimentos odontológicos assistenciais necessários, nesta avaliação.

0 presente estudo foi aprovado pelo Comitê de Ética em Pesquisa com Seres Humanos da Faculdade de Medicina da Universidade Federal de Pelotas, sob o $\mathrm{n}^{0} 077 / 09$.

\section{Resultados}

A rede de atenção básica do município realizou 120.070 procedimentos durante 0 ano de 2008. A Tabela 1 apresenta os procedimentos ambulatoriais referentes às necessidades estudadas: restaurações, extrações e tratamentos periodontais. Na totalidade, foram realizados 47.179 procedimentos nos serviços de saúde bucal, sendo que as restaurações $(42,4 \%)$ e as raspagens supragengivais $(33,4 \%)$ foram os mais frequentes.

Na Figura 1, observam-se as retas com as intrapolações das necessidades de tratamento odontológico estimadas para o presente estudo, segundo os modelos de polinômios fracionais. Os quatro gráficos que compõem a figura mostram as grandes mudanças de necessidade com o decorrer da idade: crescentes para extrações; e decrescentes para as demais necessidades avaliadas. As necessidades também variaram em relação aos quintis de renda, principalmente para as exodontias e restaurações, inversamente proporcionais à renda. Para as necessidades periodontais, pouca diferença em relação aos estratos de renda foi observada para raspagem supragengival. As necessidades de raspagem subgengival, que se mantiveram estáveis com a idade, no estrato de maior renda, reduziram-se entre aqueles de menor renda.

As necessidades estimadas estão demonstradas na Tabela 2, que apresenta o número de indivíduos, de dentes e de sextantes com necessidade de cada tipo de procedimento. Considerando-se que um mesmo indivíduo pode necessitar de mais de um procedimento, as necessidades foram calculadas por procedimento: 274.085 elementos dentários necessitavam de restaurações; 107.659 , extrações; 282.986 sextantes, tratamento para remoção do cálculo dentário; e 17.803 sextantes necessitavam de tratamento para bolsa periodontal. As necessidades mais prevalentes entre a população de 15 anos de idade ou mais foram a raspagem periodontal supragengival $(46,0 \%)$ e os procedimentos restauradores $(39,7 \%)$. Somente para a resolução das necessidades de tratamento levantadas pelo estudo, seriam necessárias ao menos 682.533 consultas clínicas, o que representa mais de 14 vezes a produção anual registrada pelo município de Pelotas-RS em 2008. 
Tabela 1 - Produção ambulatorial por necessidades avaliadas no município de Pelotas, estado do Rio Grande do Sul. Brasil, 2009

\begin{tabular}{lcc}
\hline \multirow{2}{*}{ Procedimentos } & \multicolumn{2}{c}{ Produção Ambulatorial da Rede Básica } \\
\cline { 2 - 3 } & $\mathbf{n}$ & $\%$ \\
\hline Restaurações $^{\mathrm{a}}$ & 20.022 & 42,4 \\
Extrações $^{\mathrm{a}}$ & 6.052 & 12,8 \\
Raspagem, Alisamento e Polimento Supragengival $^{\mathrm{b}}$ & 15.740 & 33,4 \\
Raspagem e AlisamentoSubgengival $^{\mathrm{b}}$ & 2.811 & 6,0 \\
Raspagemcorono-radicular $^{\mathrm{T}}$ & 2.554 & 5,4 \\
\hline TOTAL & $\mathbf{4 7 . 1 7 9}$ & $\mathbf{1 0 0 , 0}$ \\
\hline
\end{tabular}

a) Procedimentos realizados em dentição permanente

b) Procedimentos realizados por sextantes

Fonte:Sistema de Informaçōes Ambulatoriais do Sistema Único de Saúde (SIA/SUS), 2008.

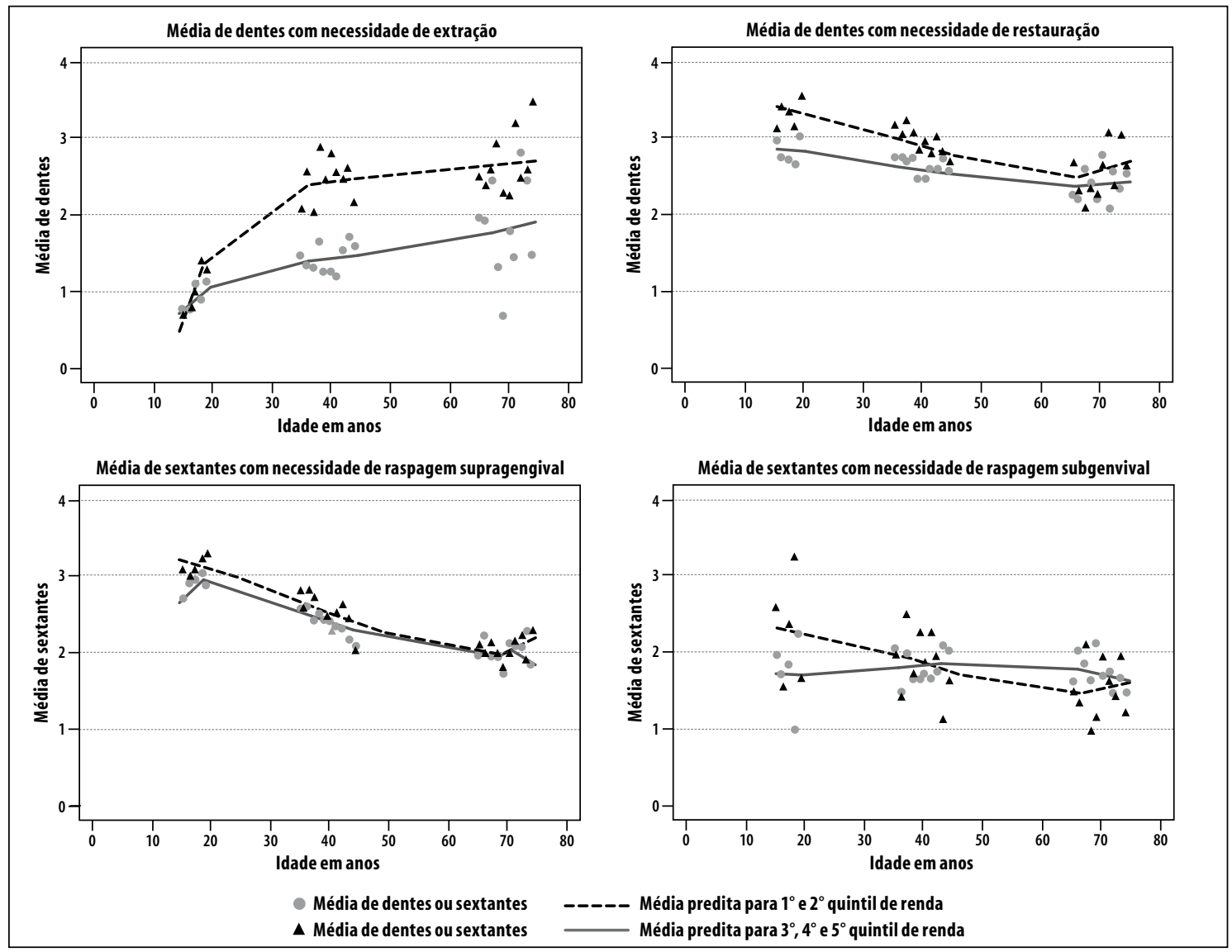

Figura 1 - Necessidades de tratamento odontológico estimadas para indivíduos com 15 anos de idade ou mais, segundo os Modelos de Regressão de Polinômios Fracionais. Brasil, Inquérito de Saúde Bucal 2003

\section{Discussão}

Os dados aqui apresentados apontaram a magnitude das necessidades de tratamento odontológico da população de Pelotas-RS, que ultrapassa, largamente, o potencial de atendimento clínico oferecido pelo serviço de Saúde Bucal.

Entre as limitações deste estudo, encontra-se a exclusão dos procedimentos realizados em dentição permanente para a população abaixo de 15 anos 
Tabela 2 - Necessidades em saúde bucal da população de 15 anos de idade ou mais no município de Pelotas, estado do Rio Grande do Sul, a partir de estimativa baseada no Inquérito de Saúde Bucal 2003. Brasil, 2009

\begin{tabular}{|c|c|c|c|c|}
\hline Procedimentos & № de indivíduos & $\%$ & $\mathrm{~N}^{0}$ de dentes & $\mathrm{N}^{0}$ de sextantes \\
\hline Restauração & 93.158 & 39,7 & 274.085 & - \\
\hline Extração & 37.004 & 15,7 & 107.659 & - \\
\hline Raspagem Periodontal Supragengival ${ }^{\mathrm{a}}$ & 107.961 & 46,0 & - & 282.986 \\
\hline Raspagem Periodontal Subgengival ${ }^{b}$ & 9.598 & 4,0 & - & 17.803 \\
\hline Total com necessidade & 234.594 & 100,0 & 381.744 & 300.789 \\
\hline
\end{tabular}

de idade. Outra limitação refere-se à não inclusão, nas estimativas, das necessidades de tratamentos pulpares e reabilitações com próteses, por falta de parâmetros para o cálculo do número de consultas: a Saúde Bucal do município não oferece esses serviços. Contudo, mais do que identificar o número exato de necessidades e consultas assistenciais necessárias para resolução dos problemas de saúde bucal da população, este trabalho demonstra que um modelo de atenção à saúde voltado exclusivamente ao atendimento clínico acaba por acumular enormes necessidades de tratamento. Não foram identificados na literatura acadêmica outros estudos com o objetivo de avaliar o déficit do potencial produtivo em relação às necessidades de tratamento odontológico, o que denota a originalidade do trabalho, embora limite a comparação dos resultados encontrados.

Este trabalho também evidenciou as acentuadas diferenças nas necessidades de tratamento por estratos de renda, evidenciando um quadro de iniquidades dos agravos bucais. A relação entre doenças bucais e iniquidades de renda pode ser explicada por diferenças nos comportamentos em saúde e acesso a serviços. Indivíduos com piores condições socioeconômicas possuem maior prevalência de tabagismo, ${ }^{19}$ consomem maior quantidade de alimentos doces, ${ }^{20}$ reproduzem piores padrões de higiene bucal ${ }^{21}$ e acessam ou utilizam com menor frequência os serviços de saúde preventivos e assistenciais. ${ }^{22,23} \mathrm{Em}$ Pelotas-RS, a proporção de adultos que utilizaram os serviços de saúde bucal no ano anterior foi de $66,2 \%$ no grupo de indivíduos com maior renda, frente a $41,9 \%$ no grupo de indivíduos com menor renda. ${ }^{22}$
Estudo sobre o uso dos serviços públicos odontológicos no Município revelou que a população de menor renda fazia mais uso desses serviços, ${ }^{23}$ resultado consistente com estudo realizado a partir de dados do SB Brasil 2003. ${ }^{24}$ Estes estudos também identificaram que os usuários dos serviços públicos tiveram maior probabilidade de realizar essa consulta motivados por algum problema, quando comparados àqueles que utilizaram os mesmos serviços de forma regular ou preventiva. Camargo e colaboradores ${ }^{23}$ apontam que $80,0 \%$ dos usuários procuraram pelo serviço odontológico público de Pelotas-RS em decorrência de um problema instalado.

Sendo a população de menor renda a que apresenta maior necessidade acumulada de tratamento odontológico, é compreensível que os serviços públicos vejam-se pressionados a dedicar grande parcela de sua capacidade produtiva para resolver problemas pontuais. Por não lograr reduzir a incidência de problemas mediante ações preventivas, essa estratégia de resposta à pressão imediata dos usuários leva a um aumento das necessidades de tratamento no médio e no longo prazos, até o ponto em que o sistema não é mais capaz de atender a demanda. Estudo realizado no Rio Grande do Sul identificou que moradores dos municípios com maiores taxas de procedimentos individuais preventivos tiveram menor chance de apresentar cárie não tratada, quando comparados aos habitantes dos municípios que apresentavam as menores taxas desses procedimentos, sinalizando que o serviço público pode ter contribuído para a diminuição do número de cáries não tratadas. ${ }^{25} \mathrm{Não}$ é tarefa fácil organizar um serviço que contemple tanto 
ações preventivas quanto uma oferta assistencial que estimule sua utilização de forma regular. Não obstante, a busca desse equilíbrio é necessária.

0 presente estudo demonstrou um aumento das necessidades de exodontia proporcional à idade, o que pode refletir a não exposição da população adulta aos benefícios do flúor no decurso de sua vida. Daí viria 0 maior acúmulo de cárie, suas sequelas e consequente necessidade de perda dentária, conforme observaram Peres e colaboradores ${ }^{26}$ e Narvai e colaboradore ${ }^{27} 0$ aumento das necessidades de exodontia acaba por ser inversamente proporcional às necessidades restauradoras, uma vez que o edentulismo aumenta com a idade. No Brasil, a proporção de pelo menos um dente perdido devido à cárie foi de 38,9\% em adolescentes de 15 a 19 anos, ${ }^{28}$ de $55,0 \%$ nos adultos entre 35 e 44 anos $^{3}$ e chegou a $92,9 \%$ no grupo dos idosos, entre 65 e 74 anos de idade. ${ }^{12}$ Barbato e colaboradores ${ }^{3}$ também encontraram que a redução das necessidades periodontais aumentava com a idade e nos estratos de menor renda. Essa redução pode ser explicada pelo aumento progressivo da perda dentária nesses grupos. ${ }^{3}$

No Brasil, os modelos de assistência, em muitos casos, foram implementados pela simples cópia do modelo da prática privada e sua aplicação ao setor público, com enfoque individual e curativo-reparador, sem utilizar informações epidemiológicas que subsi-

\section{Referências}

1. World Health Organization. The world oral health report 2003: continuous improvement of oral health in the 21st century - the approach of the WHO Global Oral Health Programme. Geneva: World Health Organization; 2003.

2. Nickel DA, Lima FG, Bidigaray da Silva B. Modelos assistenciais em saúde bucal no Brasil. Cadernos de Saúde Pública. 2008; 24(2):241-246.

3. Barbato PR, Nagano HCM, Zanchet FN, Boing AF, Peres MA. Perdas dentárias e fatores sociais, demográficos e de serviços associados em adultos brasileiros: uma análise dos dados do Estudo Epidemiológico Nacional (Projeto SB Brasil 2002-2003). Cadernos de Saúde Pública. 2007; 23(8):1803-1814.

4. Ministério da Saúde. Secretaria de Atenção à Saúde. Diretrizes da política nacional de saúde bucal. Brasília: Ministério da Saúde; 2004. diassem o planejamento da assistência. ${ }^{29} \mathrm{~A}$ alternativa que se apresenta viável, especialmente por seu conhecido potencial preventivo, é a de um modelo que enfatize ações de promoção e prevenção em saúde bucal tendo como meta a redução da incidência das respectivas patologias. A médio prazo, essa estratégia - se implementada de forma efetiva - poderá resultar na redução das necessidades clínicas e consequente diminuição da pressão da demanda sobre o serviço. ${ }^{5}$

0 município de Pelotas-RS oferece assistência, não atua na prevenção das doenças bucais. Assim, as necessidades de tratamento tornam-se extremamente elevadas e geram alto custo para a sociedade, não apenas no aspecto financeiro como no sofrimento gerado pelas doenças bucais. Completando o quadro de inadequação, fica evidente que o potencial produtivo do serviço odontológico de Pelotas-RS está muito aquém de atender a todas as necessidades da população.

\section{Contribuição dos autores}

Jaccottet CMG desenvolveu a hipótese do estudo, analisou e interpretou os dados e redigiu a primeira versão do manuscrito.

Barros AJD, Camargo MBJ e Cascaes AM analisaram e interpretaram os resultados e realizaram a revisão crítica do artigo.

5. Antunes JLF, Narvai PC. Políticas de saúde bucal no Brasil e seu impacto sobre as desigualdades em saúde. Revista de Saúde Pública.

2010; 44(2):360-365.

6. Frazão P, Narvai PC. Saúde bucal no Sistema Único de Saúde: 20 anos de lutas por uma política pública. Saúde em Debate. 2009; 33(81):64-71.

7. Ministério da Saúde. Departamento de Atenção Básica. Números da Saúde da Família [Internet]. Brasília: Ministério da Saúde; 2011 [acessado durante o ano de 2011]. Disponível em http://dab.saude.gov. br/abnumeros.php\#numeros.

8. Pinheiro RS, Torres TGZ. Uso de serviços odontológicos entre os Estados do Brasil. Ciência \& Saúde Coletiva. 2006; 11(4):999-1010.

9. Barros SG, Chaves SCL. A utilização do Sistema de Informações Ambulatoriais (SIA-SUS) como 
instrumento para caracterização das ações de saúde bucal. Epidemiologia e Serviços de Saúde. 2003; 12(1):41-51.

10. Barros AJD, Bertoldi AD. Desigualdades na utilização e no acesso a serviços odontológicos: uma avaliação em nível nacional. Ciência \& Saúde Coletiva. 2002; 7(4):709-717.

11. Instituto Brasileiro de Geografia e Estatística. Censo brasileiro de 2010 [Internet]. Rio de janeiro: Instituto Brasileiro de Geografia e Estatística; 2011 [acessado durante o ano de 2011]. Disponível em: http://www.ibge.gov.br/cidadesat/painel/painel. php?codmun $=431440$

12. Ministério da Saúde. Secretaria de Atenção à Saúde. Departamento de Atenção Básica. Projeto SB Brasil 2003: condições de saúde bucal da população brasileira 2002-2003: resultados principais. Brasília: Ministério da Saúde; 2004. (Série C. Projetos, Programas e Relatórios).

13. Sheiham A, Netuveli GS. Periodontal diseases in Europe. Periodontology 2000. 2002; 29:104-121.

14. Agência Nacional de Saúde Suplementar. Dados e indicadores do setor. Taxa de cobertura de serviços [ Internet]. Brasília: Agencia Nacional de Saúde Suplementar; 2009 [acessado durante o ano de 2009]. Disponível em: http://anstabnet.ans.gov.br/ tabcgi.exe?dados/TABNET_TX.def

15. Royston P, Sauerbrei W. Multivariable model-building: a pragmatic approach to regression analysis based on fractional polynomials for modelling continuous variables. New York: Jon Wiley \& Sons; 2008.

16. Instituto Brasileiro de Geografia e Estatística. Sistema IBGE de Recuperação Automática. Demográfico e contagem [Internet]. Rio de Janeiro: Instituto Brasileiro de Geografia e Estatística [acessado durante 0 ano de 2009]. Disponível em http://www. sidra.ibge.gov.br/.

17. Ministério da Saúde. Secretaria de Atenção à Saúde. Cadastro Nacional dos Estabelecimentos de Saúde do Brasil. Relatórios e consultas [Internet]. Brasília: Ministério da Saúde; 2009 [acessado durante o ano de 2009]. Disponível em: http://cnes.datasus.gov.br/.

18. Ministério da Saúde. Departamento Nacional de auditoria do SUS. Orientações para proceder auditoria na Atenção Básica. Brasília: Ministério da Saúde; 2004.

19. Barros AJ, Cascaes AM, Wehrmeister FC, MartinezMesa J, Menezes AM. Tabagismo no Brasil: desigualdades regionais e prevalência segundo características ocupacionais. Ciência e Saúde Coletiva. 2011; 16(9):3707-3716.

20. Vinholes DB, Assuncao MC, Neutzling MB. Frequência de hábitos saudáveis de alimentação medidos a partir dos 10 Passos da alimentação saudável do Ministério da Saúde: Pelotas, Rio Grande do Sul, Brasil. Cadernos de Saúde Pública. 2009; 25(4):791-799.

21. Peres KG, Peres MA, Demarco FF, Tarquinio SB, Horta BL, Gigante DP. Oral health studies in the 1982 Pelotas (Brazil) birth cohort: methodology and principal results at 15 and 24 years of age. Cadernos de Saúde Pública. 2011; 27(8):1569-1580.

22. Peres MA, Peres KG, Thomson WM, Broadbent JM, Gigante DP, Horta BL. The influence of family income trajectories from birth to adulthood on adult oral health: findings from the 1982 Pelotas birth cohort. American Journal of Public Health. 2011; 101(4):730-736

23. Camargo MBJ, Dumith SC, Barros AJD. Uso regular de serviços odontológicos entre adultos: padrões de utilização e tipos de serviços. Cadernos de Saúde Pública. 2009; 25(9):1894-1906.

24. Pinto RS, Matos DL, Loyola Filho AI. Características associadas ao uso de serviços odontológicos públicos pela população adulta brasileira. Ciência \& Saúde Coletiva. 2012; 17(2):531-544.

25. Celeste RK, Nadanovsky P, Leon AP. Associação entre procedimentos preventivos no serviÇo público de odontologia e a prevalência de cárie dentária. Rev SaÚde Pública. 2007; 41(5):830-838.

26. Peres MA, Antunes JL, Peres KG. Is water fluoridation effective in reducing inequalities in dental caries distribution in developing countries? Recent findings from Brazil. Sozial- und Präventivmedizin. 2006; 51(5):302-310.

27. Narvai PC, Frazao P, Roncalli AG, Antunes JL. Carie dentária no Brasil: declínio, polarização, iniquidade e exclusão social. Revista Panamericana de Salud Publica. 2006; 19(6):385-393.

28. Barbato PR, Peres MA. Perdas dentárias em adolescentes brasileiros e fatores associados: estudo de base populacional. Revista de Saúde Pública. 2009; 43(1):13-25.

29. Narvai PC. Odontologia e saúde bucal coletiva. São Paulo: Hucitec; 1994.

Recebido em 28/06/2011

Aprovado em 25/05/2012 